\title{
Metro de Washington
}

\section{EE.UU.}

HARRY WEESE y asociados, arquitectos

526-7

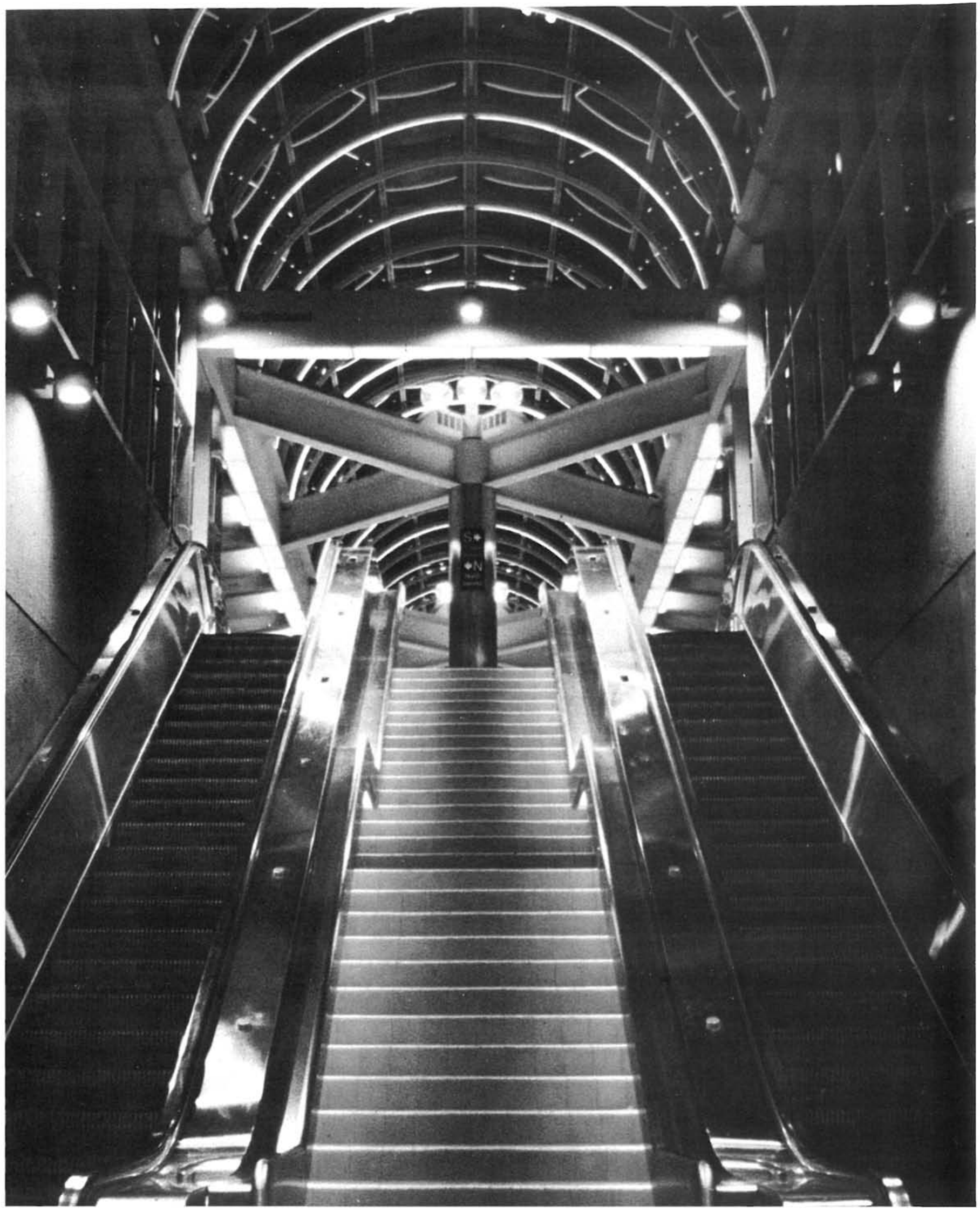


sinopsis

Se describen en este articulo los trabajos de la primera fase del Metro de Washington que, iniciado en el año 1969 será, a su terminación en el año 1983, el más moderno sistema de ferrocarril metropolitano de Norte Amériç

Es de doble carril y servirá para tres millones de usuarios.

Se han empleado distintos sistemas de obra en su realización: túnel artificial realizado mediante una zanja que después se cubre: túnel perforado, y estructuras aéreas.

Las caracteristicas de las estaciones responden a las mayores exigencias, pues tienen circuito cerrado de televisión, aire acondicionado, sistemas para atenuar el ruido, accesos especiales, sistema de protección contra el fuego y control automático del Metro.

Se estudian de un modo particular: los dos puentes sobre el Pentágono y el Anacostia, señalando sus diferencias y la estructura aérea del Aeropuerto Nacional.

Adopted March 1968, As Fevised
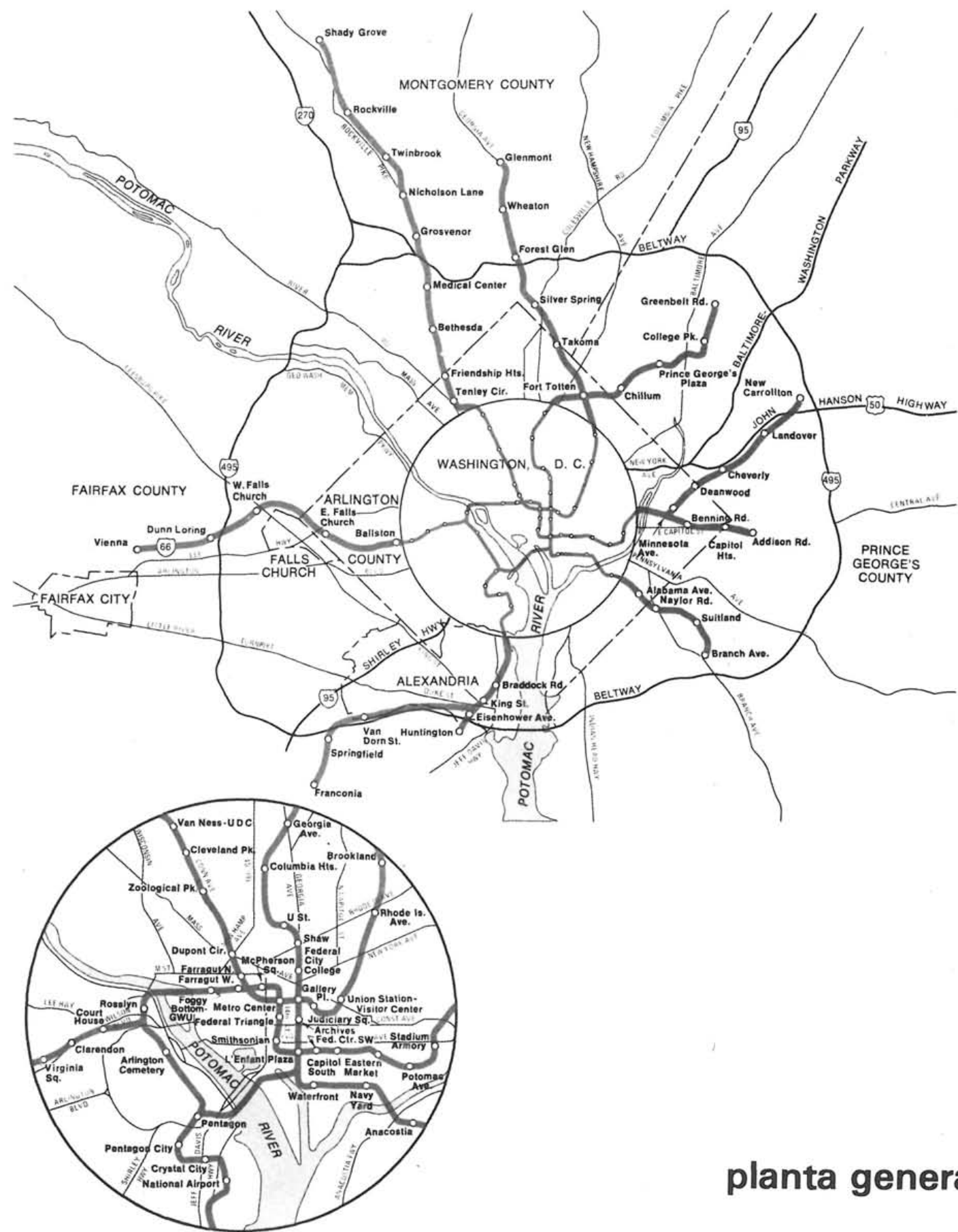

planta general 
El «Washington Post» en su edición del domingo, 5 de diciembre de 1909, publicó un artículo que ocupaba toda una página describiendo detalladamente un sistema de ferrocarril subterráneo para Washington, D.C.

En 1969 se inició la construcción en la Capital del primero de estos sistemas.

Aunque la operación comercial de la fase I se inició este año, la fecha de la realización del sistema total del Metro está fijada para finales de 1983.

El más moderno sistema de transporte rápido por ferrocarril de Norte América, el Metro de Washington, comenzó a funcionar el 29 de marzo de 1977. El mayor proyecto de obras públicas de este tipo emprendido hasta ahora en una área metropolitana de América es el Metro, de un doble carril, que sirve para cerca de tres millones de usuarios en el distrito de Columbia, la cercana Virginia y suburbios de Maryland.

Esta fase I del Metro, que cruza el Distrito Central de Washington, D.C., es de considerable interés porque consta de todos los tipos de obra usados en el sistema: túnel artificial, hecho mediante una zanja que después se cubre; túnel perforado, y estructuras aéreas. Por lo menos la mitad del sistema, 42 millas $(67,6 \mathrm{~km})$, correrá sobre la superficie.

Aunque el tramo de apertura del Metro es relativamente corto en relación al total, proporciona un enlace verdadero entre el nordeste y noroeste de Washington. Sirvió también a WHATA para probar totalmente los procedimientos y técnicas de operación a realizar en tramos mayores.

En el nordeste están los dos puntos del Metro que registran mayor afluencia de enlaces de ida y vuelta, el pasillo de la Avenida de Rhode Island para enlaces de autobuses y automóviles y la Unión Station para enlaces de ferrocarril. En el noroeste, el sistema proporciona acceso cómodo a las calles comerciales F y G, y al área comercial de la Avenida Connecticut.

En el punto medio del recorrido está la Estación de la Plaza Judicial, que sirve de acceso a los tribunales federales y municipales. El Metro ha servido para reducir el viaje de 30 minutos a una experiencia agradable de sólo 9 minutos.

Las operaciones iniciales se han realizado con un programa más conservador que el propuesto para el sistema total.

El tráfico de pasajeros se ha limitado desde las 6 de la mañana hasta las 8 de la tarde. Los trenes de cuatro vagones pasan cada 5 minutos en el período de mayor movimiento, mientras que los trenes de dos vagones lo hacen durante los demás períodos.

El andén de las estaciones estándar de 600 pies $(183 \mathrm{~m})$, puede acomodar hasta trenes de ocho vagones. La circulación en el tramo inicial de 4,6 millas $(7,4 \mathrm{~km})$ ha sido de cerca de tres veces la prevista, con un promedio diario de 23.000 pasajeros. El pasajero, un millón, recibió la bienvenida a bordo de un vagón, exactamente, dos meses después del día de apertura.

El precio del pasaje, entre las dos estaciones del Metro e incluyendo transbordo a los autobuses dentro del Distrito de Columbia es de 0,55 dólares durante las horas de mayor movimiento de la mañana y de la tarde, y 0,40 durante el resto del tiempo.

Un gran aumento de la circulación se produjo entre el distrito de negocios, alrededor de la Estación del Norte de Farragut, y el área comercial del Centro durante el tiempo del almuerzo.

La Autoridad respondió rápidamente a la demanda, incluyendo trenes de cuatro vagones cada 5 minutos.

Se han usado diferentes métodos de construcción, desde el túnel artificial que después se cubre y las sofisticadas máquinas para roca dura, al acero moderno y hormigonado postesado de las estructuras aéreas.

La arquitectura del Metro armoniza y complementa con la arquitectura monumental de la capital de la Nación.

La bóveda de 30 pies $(9,1 \mathrm{~m})$ está situada sobre el plano de la vía, con superficie de hormigón moldeado in situ o pretensado. 

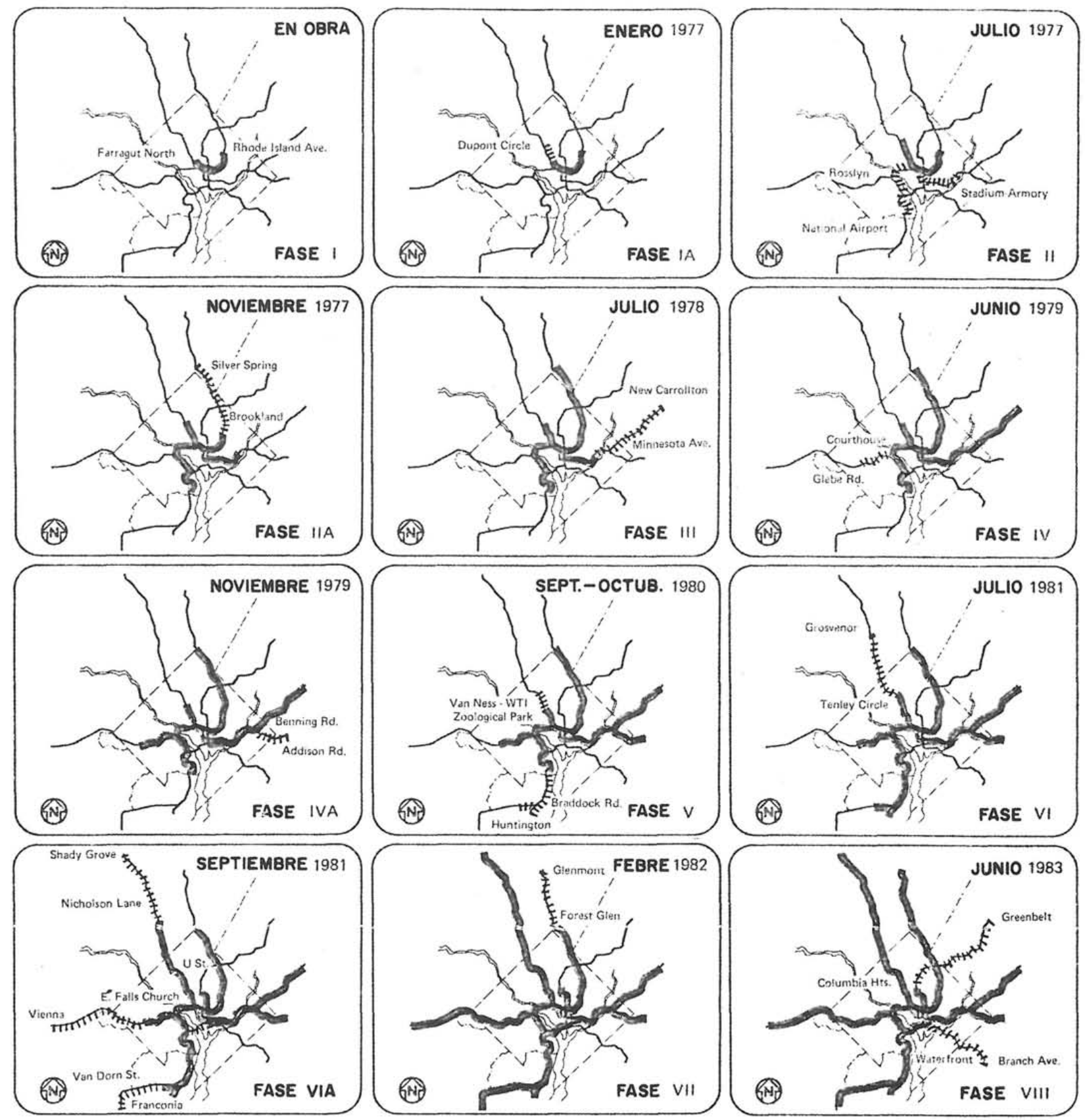

fases de construcción

Se prestó gran atención a la impermeabilización de las superficies de hormigón expuestas, usándose extensamente paneles de bentonita Volday en el exterior. Este tipo de estructura, además de ser estéticamente agrardable, sirve para dominar el ruido.

Las estaciones tienen circuito cerrado de televisión. El alumbrado a lo largo del sistema es indirecto y las pareces tienen dificultad para agrarrarse, impidiendo el vandalismo y las pintadas.

Las estaciones tienen aire acondicionado para contrarrestar en los veranos húmedos el aire caliente de Washington; pero la calefacción no es necesaria, ya que se produce suficiente calor durante las operaciones del tren manteniéndose una temperatura confortable.

También se prestó gran atención al control del ruido y la vibración; las técnicas usadas incluyen carriles soldados de manera continua, pasadores de vía resistentes al choque, losas de vía flotante, apoyo cuidadoso de la rueda y especificaciones del vehículo convincentes. El Metro es el primer sistema de transporte donde se hace bastante uso de las losas de hormigón flotante para reducir el ruido y la vibración en las proximidades de la vía.

Bajo el voladizo del borde del andén, bien sea del tipo central o lateral, se adosan paneles absorbentes del sonido reduciendo, en gran parte, el nivel de ruido del ambiente en las estaciones. 
Los vagones están apoyados sobre chorros de aire que amortiguan la vibración transmitida a las vías. La puerta del vagón se mantiene dentro de las 0,25 pulgadas $(6 \mathrm{~mm})$ del borde del andén, tanto durante la inflación como con la defleción del aire. Como resultado de estas medidas, el vehículo entra y sale de una manera muy elegante de las estaciones, con mucho menos ruido que el normal en los sistemas de transporte rápido.

Se ha tenido en gran consideración la seguridad del público, tanto ante accidentes como contra el crimen, consiguiendo a la vez un confortable medio ambiente. Las escaleras mecánicas, el aire acondicionado, la comunicación instantánea con la policía del Metro, así como con los departamentos locales contribuyen a la alta calidad del sistema de transporte.

Desde el día de apertura los gráficos de circulación indican que el uso de accesos especiales para inválidos, etc. ha sido bajo.

Han sido extensas las precauciones contra el fuego, incluyendo aparatos de lucha completa contra el fuego, equipos de detección del fuego y del humo, baterías de emergencia de poderoso alumbrado y túnel contra el fuego que puede liberar agua cada 200 pies $(61 \mathrm{~m})$. En una emergencia, el sistema de detección del fuego y del humo, abrirá automáticamente las puertas cerradas, parará las escaleras mecánicas de regreso y abrirá las puertas de los usuarios para, mediante la «apertura libre», conseguir la salida rápida.

\section{Control automático del tren}

Los trenes funcionan de manera totalmente automática desde que fue inaugurado el Metro, el 29 de marzo. Cada tren tiene un solo mando que controla las puertas de los vagones y actúa en la puesta en marcha de los trenes.

El sistema automático de control está compuesto por tres subsistemas: Supervisión Automática del Tren (ATS); Operaciones Automáticas del Tren (ATO) y Protección Automática del Tren (ATP).

El sistema ATS usa información, por medio de computadores, que "avisa» a los vehículos del Metro: del tiempo entre estaciones, distancia entre vehículos, tiempo de estancia en las estaciones y otras características necesarias para el funcionamiento de los vehículos. Es un sistema de gran potencia.
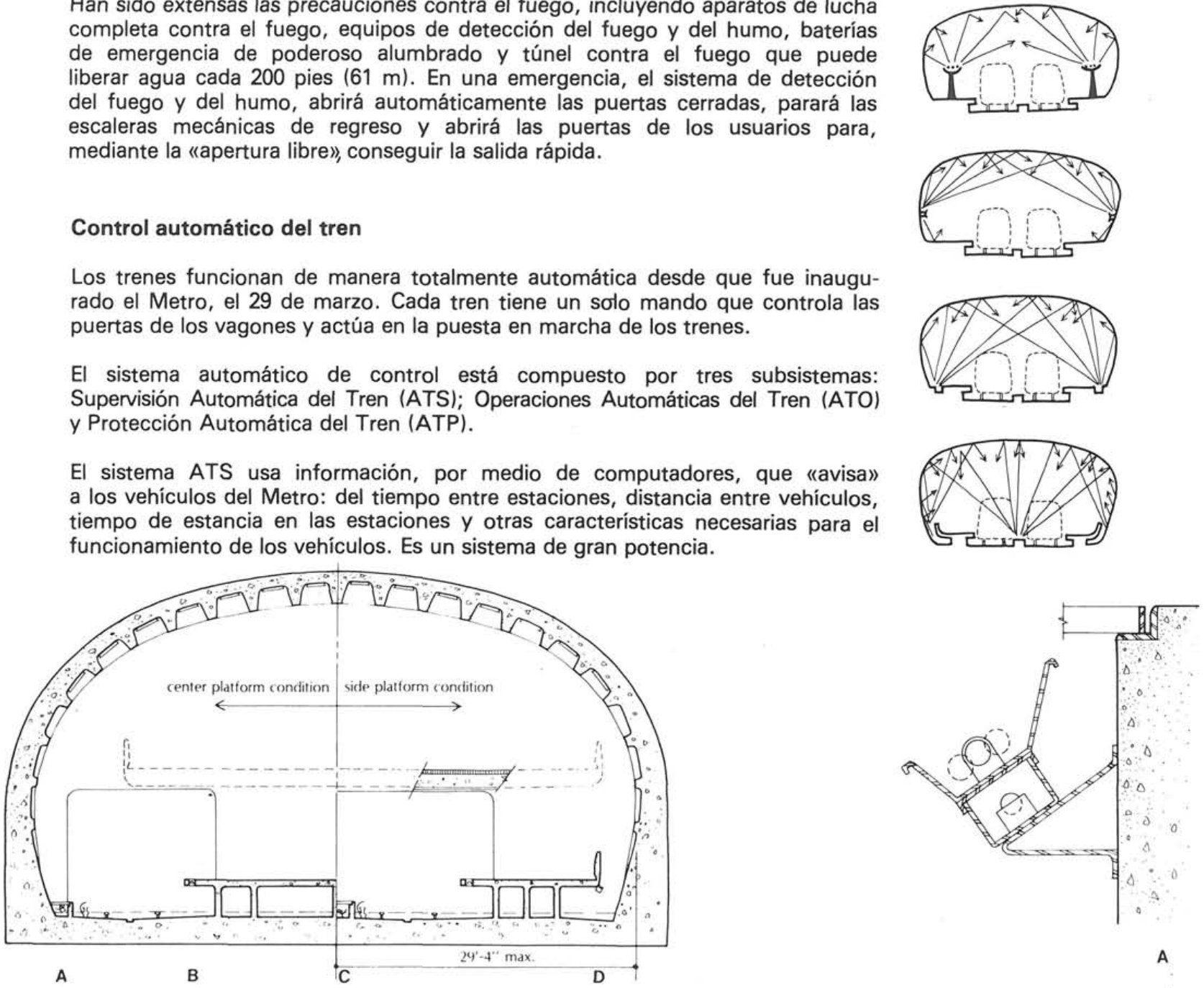

A

\section{iluminación}
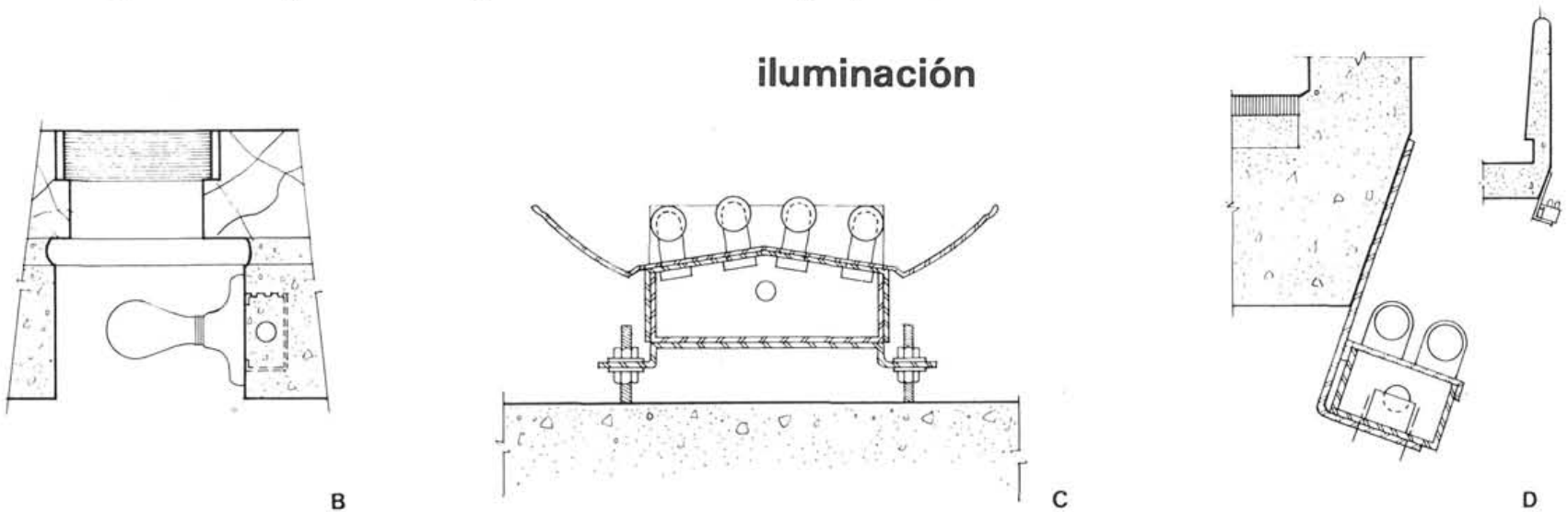


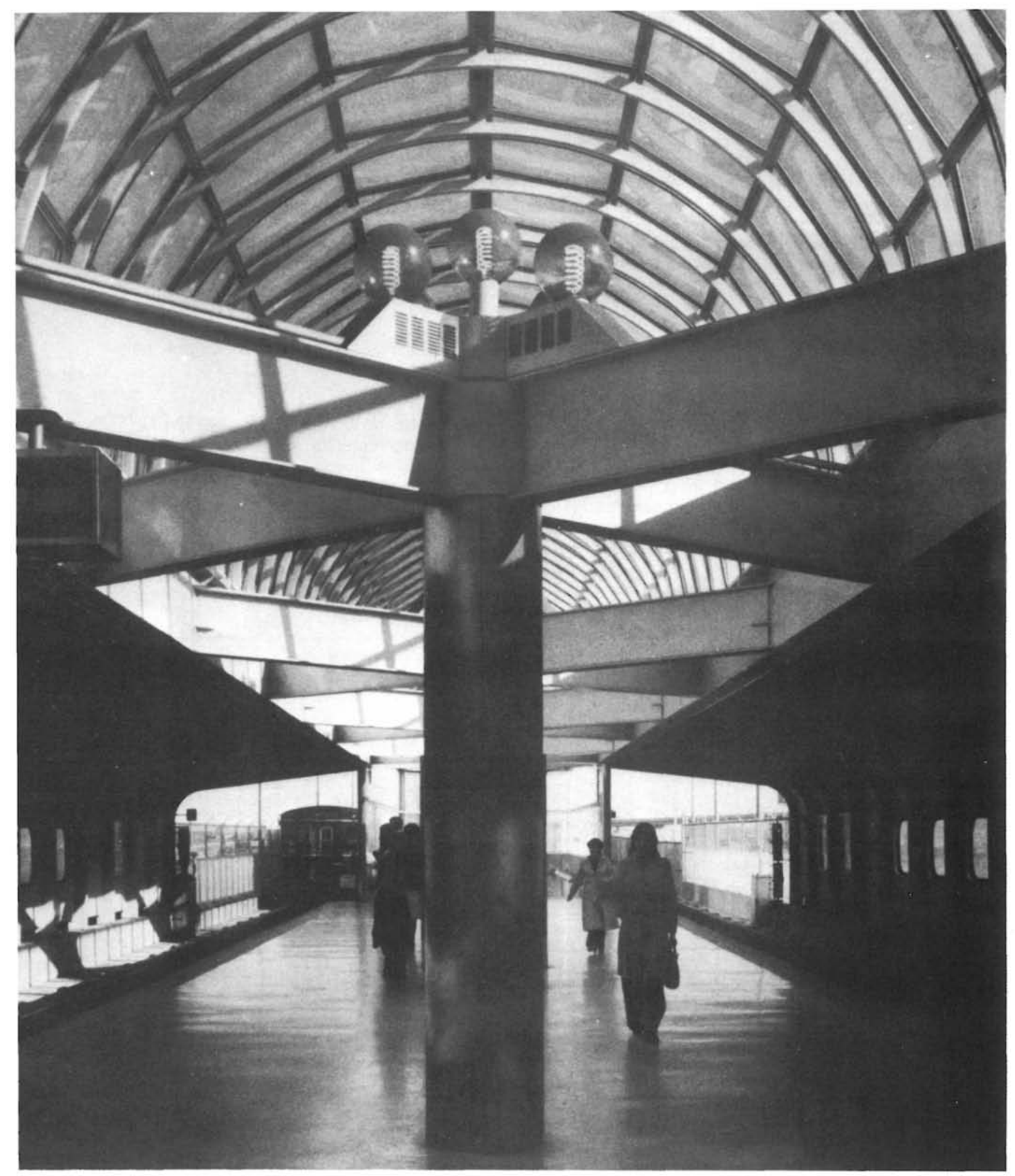

EI ATO convierte las señales del ATS en instrucciones de operaciones para los trenes, pero antes de que esas instrucciones sean transmitidas, son evaluadas por el tercer subsistema, el ATP. El ATP pasa por encima de los otros dos sistemas y rectifica algunas órdenes no muy claras. Pone los límites de velocidad para los trenes, si es necesario, y ejerce otras restricciones en aras de la seguridad.

Ei Sistema de Control Automático del tren está proyectado como un sistema de seguridad para que cualquier mal funcionamiento termine en una situación segura. Si es necesario, el conductor puede asumir el control manual, pero incluso bajo este tipo de control la protección de sobrevelocidad es aprovechada.

Para resolver los problemas de arquitectura existentes en un proyecto de este tipo se seleccionó a Harry Weese y Asociados. 
El primer contrato comprendía cuatro trabajos:

1) Abrir una oficina en Washington.

2) Coordinar el trabajo arquitectónico con De Lew, Cather y Compañía, Consulting General de Ingeniería, quienes ya estaban contratados desde diciembre de 1965.

3) Realizar una investigación completa de 18 sistemas de transporte, de diferentes países, para averiguar el estado del arte de transporte de masas concibiendo posteriormente los rasgos esenciales que se deben usar para el'Metro de Washington.

4) Recomendar a la NCTA un estilo arquitectónico para todos los elementos del ferrocarril.

Poco antes de firmar el contrato, el presidente Johnson escribió a McCarter para que se proyectara y construyera el mejor sistema posible de transporte. Este interés del presidente de los Estados Unidos nos habla de la necesidad de la calidad para este importante servicio.

Harry Weese, Robert Reynolds, jefe de proyectos y Stanley Allan, director de proyecto, visitaron Lisboa, Madrid, Barcelona, Roma, Milán, Viena, Francfort, Berlín, Hamburgo, Londres, París, Oslo, Estocolmo, Leningrado, Moscú, Tokio, Montreal, Toronto y Ciudad de México. Estos tres arquitectos pasaron 42 días inspeccionando los sistemas, entrevistando a los administrativos y técnicos de los transportes públicos, fotografiando cientos de vistas de los sistemas, haciendo croquis-pluma y tinta $y$, en general, familiarizándose con el arte.

El 6 de julio de 1966 se presentó la manera de concebir las estaciones. Durante el resto de 1966 y hasta septiembre de 1967 continuó el desarrollo del concepto arquitectónico con aplicaciones particulares en estaciones específicas. La Comisión de Bellas Artes dio su consentimiento en septiembre de 1967.

El camino a partir de entonces estuvo limpio para realizar un proyecto definitivo a todos los elementos del sistema, coordinando el trabajo con De Lew, Cather y Compañía, ciudadanos locales, órganos

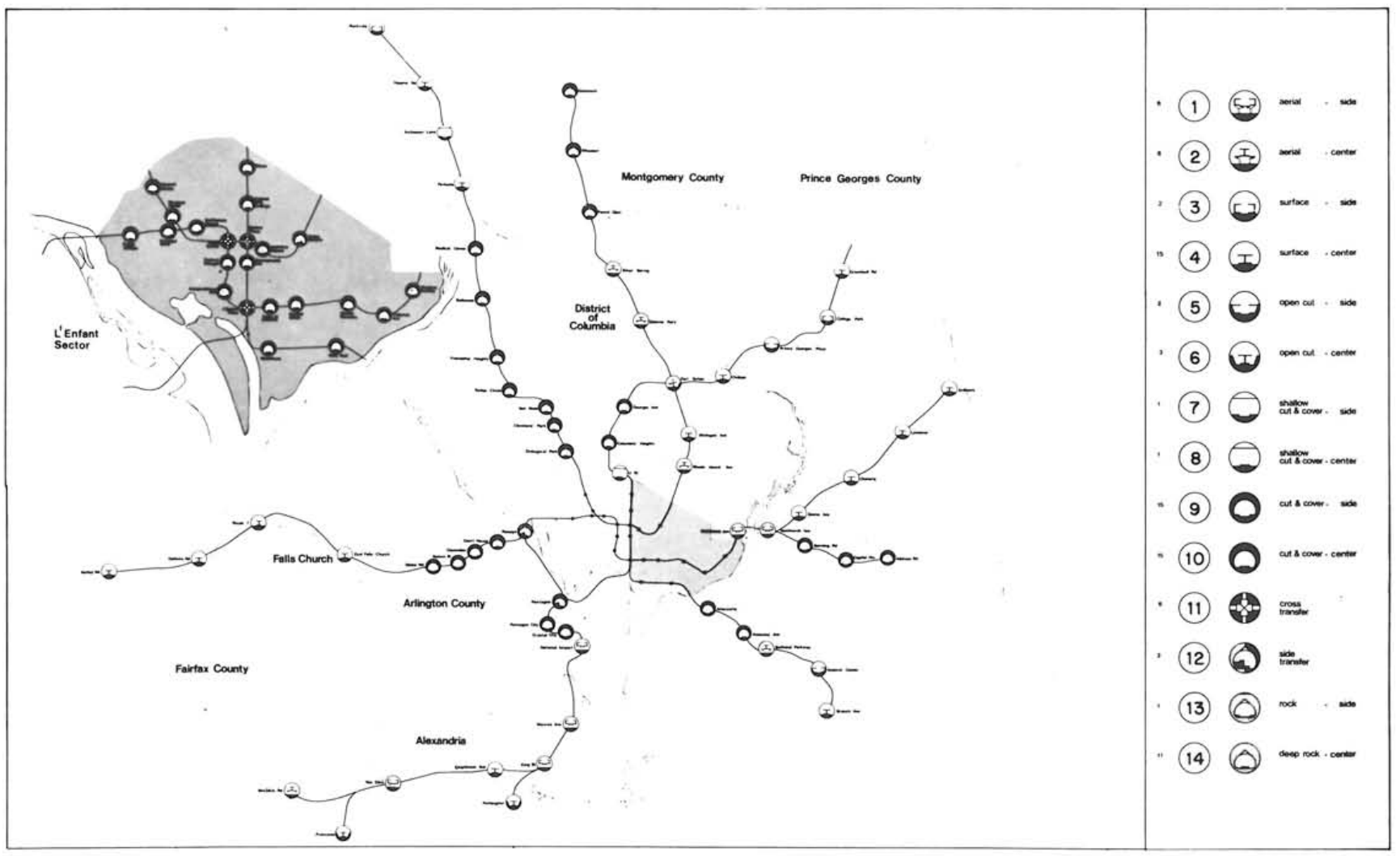

1.-Aèreo lateral. 2.-Aèreo central. 3.-Superficie lateral. 4.-Superfi cie central. 5. - Foso abierto lateral. 6.-Foso abierto central. 7.-Poco profundo. Foso cubierto lateral. 8.-Poco profundo. Foso cubierto central. 9.- Túnel lateral. 10.-Túnel central. 11.-Transbordo-cruce. 12. - Transbordo lateral. 13. - Roca lateral. 14.-Roca profunda central. 


\section{detalles}
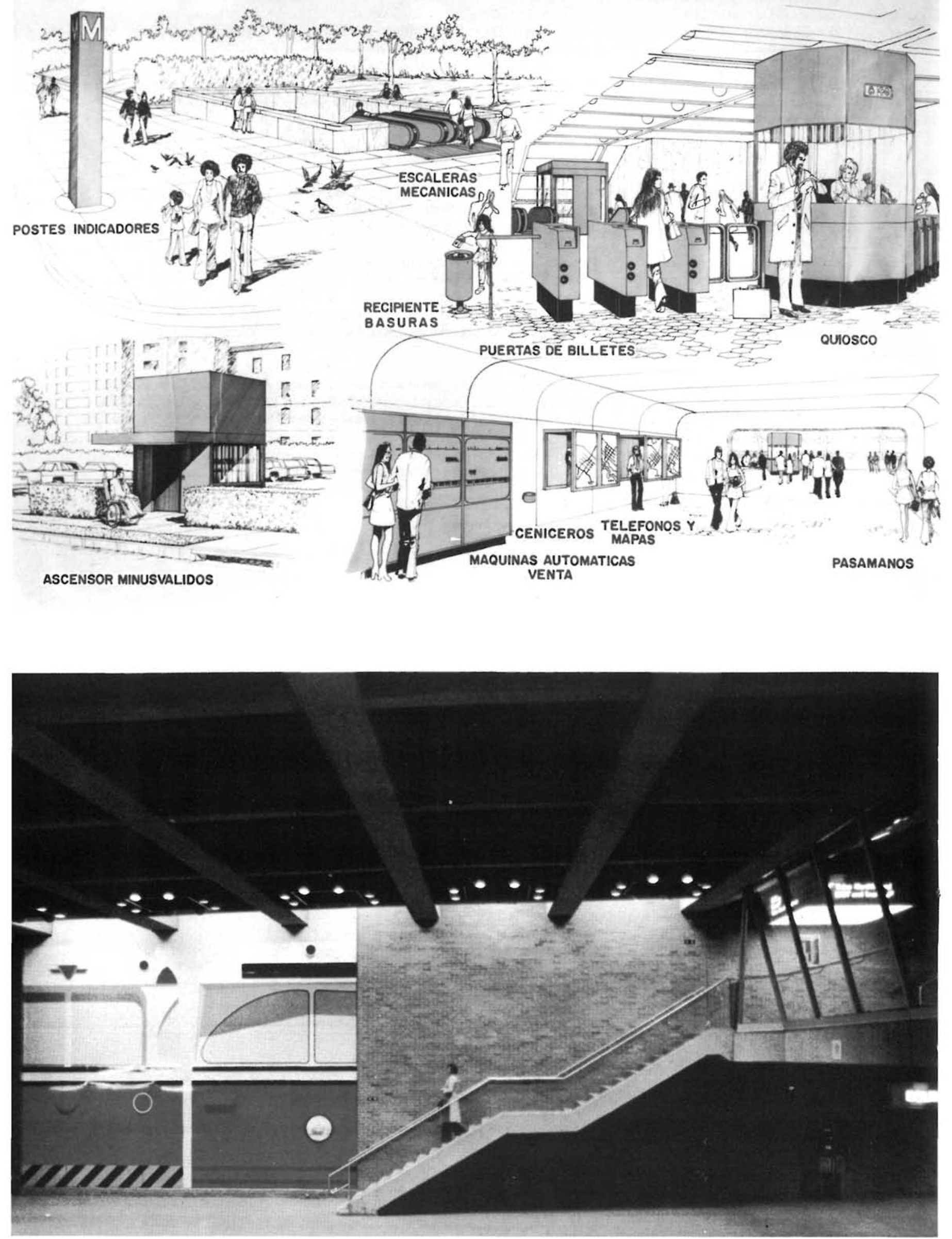


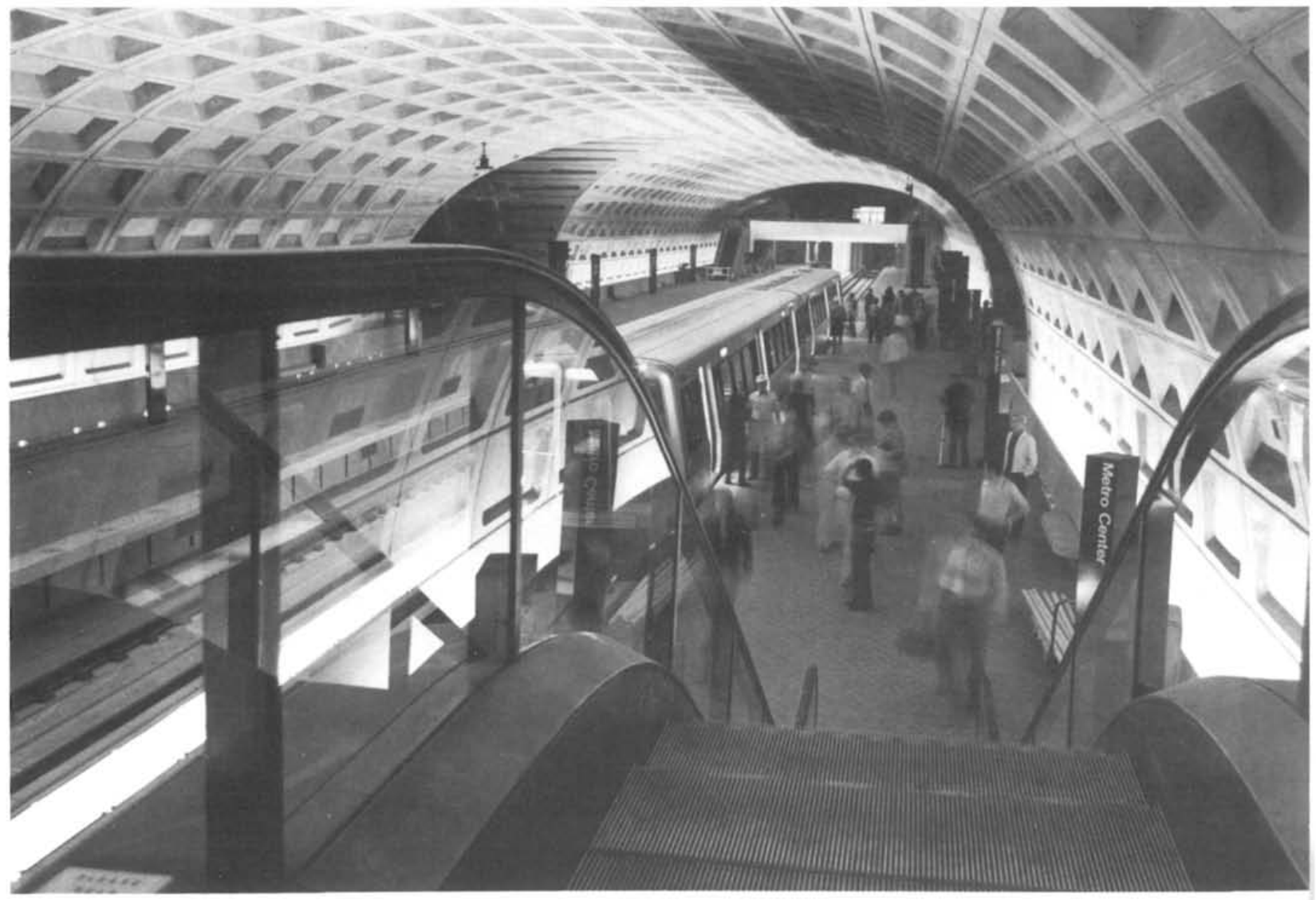

oficiales y otros. El trabajo de Harry Weese introdujo en los proyectos definitivos elementos de continuidad a lo largo del sistema-alumbrado, gráficos, reconstitución del paisaje, mobiliario, materiales, colores y protección contra el tiempo.

Además, Harry Weese y Asociados fueron contratados para sugerir ideas sobre el vehículo de transporte. Harry Weese y Stanley Allan hicieron un segundo viaje al extranjero para ponerse en contacto con Transportes de Londres y con BVG de Berlín para investigar de una manera amplia el estado del arte de los vehículos de transporte en estos dos sistemas.

En agosto de 1966, Harry Weese y Asociados presentó un diseño de vehículo al NCTA. El criterio básico para el vehículo detallado por De Lew, Cather y Compañía, fue de que los vagones tuvieran 75 pies $(22,86 \mathrm{~m})$ de longitud, 10 pies $(3,04 \mathrm{~m})$ de anchura, 10 pies $(3,04 \mathrm{~m})$ de altura y totalmente automático, con una velocidad de 75 millas $/ \mathrm{h}$. $(120 \mathrm{~km} / \mathrm{h})$. Los vagones tendrán 82 asientos, con espacio suplementario para otras 80 personas, y serán desde luego con ruedas y raíles de acero; el tercer raíl podría conectar los vehículos a 750 voltios. Harry Weese y Asociados recomendaron que los vehículos fuesen pintados de un color rojo cálido. Esto se basaba en observaciones de los colores de los vagones en Londres; el amarillo cromo en Berlín, el naranja en Milán y Ciudad de México, azul claro en Montreal y verde en Estocolmo. Su recomendación para la pintura de los vehículos se basó sobre todo en la composición de los elementos de las estaciones donde los vagones rojos podrían proporcionar un buen nivel de color, ya que las estaciones tienen el gris tostado del hormigón, el blanco del granito del borde del andén y el rojo del pavimento de piedra hexagonal en el andén, entresuelo y pasillos. La decisión, después de muchas controversias sobre la naturaleza de los vehículos y el color, fue tomada por la WMATA siguiendo la recomendación del diseñador del vehículo, tomando el aluminio natural como color para el acabado de los vagones.

\section{Túneles}

En el sistema hay aproximadamente 15 millas $(24 \mathrm{~km})$ de túneles en roca, 11 millas $(17,7 \mathrm{~km})$ de túneles de tierra y 22 millas $(35,4 \mathrm{~km})$ de túneles realizados mediante una zanja que después se cubre.

En los túneles en roca, aunque a veces se usaron métodos manuales convencionales de laboreo de minas, las limitaciones debidas a la perforación con explosivos y a los efectos de vibración hicieron que se emplearan técnicas automáticas.

Una máquina Robbins, de 900 HP y 285 t fue usada en la sección A006 bajo la Avenida de Connecticut, una de las mejores calles arteriales de la ciudad, alcanzando velocidades notables de penetración. 
Se inició la perforación de los túneles a través de un material de granito, cuarzo, hornablenda y gneis de mica, con tensiones de compresión de hasta $1.546,8 \mathrm{~kg} / \mathrm{cm}^{2}$.

Además de la máquina Robbins, se han usado otras máquinas en estos túneles: una Dresser en la sección A008 y una Jarva en la sección K001.

\section{La estructura aérea del aeropuerto nacional}

El tramo siguiente del Metro de Washington que se abrirá, proporcionará servicio al Aeropuerto Nacional en Virginia, cerca del río Potomac. Aproximadamente 1 milla $(1,6 \mathrm{~km})$ de estructura elevada conduce al Metro de la Ruta Hungtinton, a lo largo del Aeropuerto Nacional.

La estructura aérea es paralela a los caminos de acceso al aeropuerto, en la zona cercana a los edificios de la terminal, y cruza dichos accesos por el lado Sur del Aeropuerto y por la Autopista George Washington.

La estación del Metro del Aeropuerto Nacional es una estructura aérea, de doble andén, de tres carriles localizados a lo largo del lado oeste de los caminos de acceso al aeropuerto opuestos al edificio de la Terminal Norte.

Cada uno de los andenes de salida y entrada de los usuarios soporta una larga bóveda de unos 321 pies $(98 \mathrm{~m})$. Estas bóvedas son curvas moldeadas in situ y formadas por cuatro unidades estructurales de placas delgadas. Cada unidad de placa está extendida de manera continua sobre dos pilares espaciados 41,7 pies $(13 \mathrm{~m})$, con una ménsula de 20 pies $(6 \mathrm{~m})$ sobresaliendo por cada extremo.

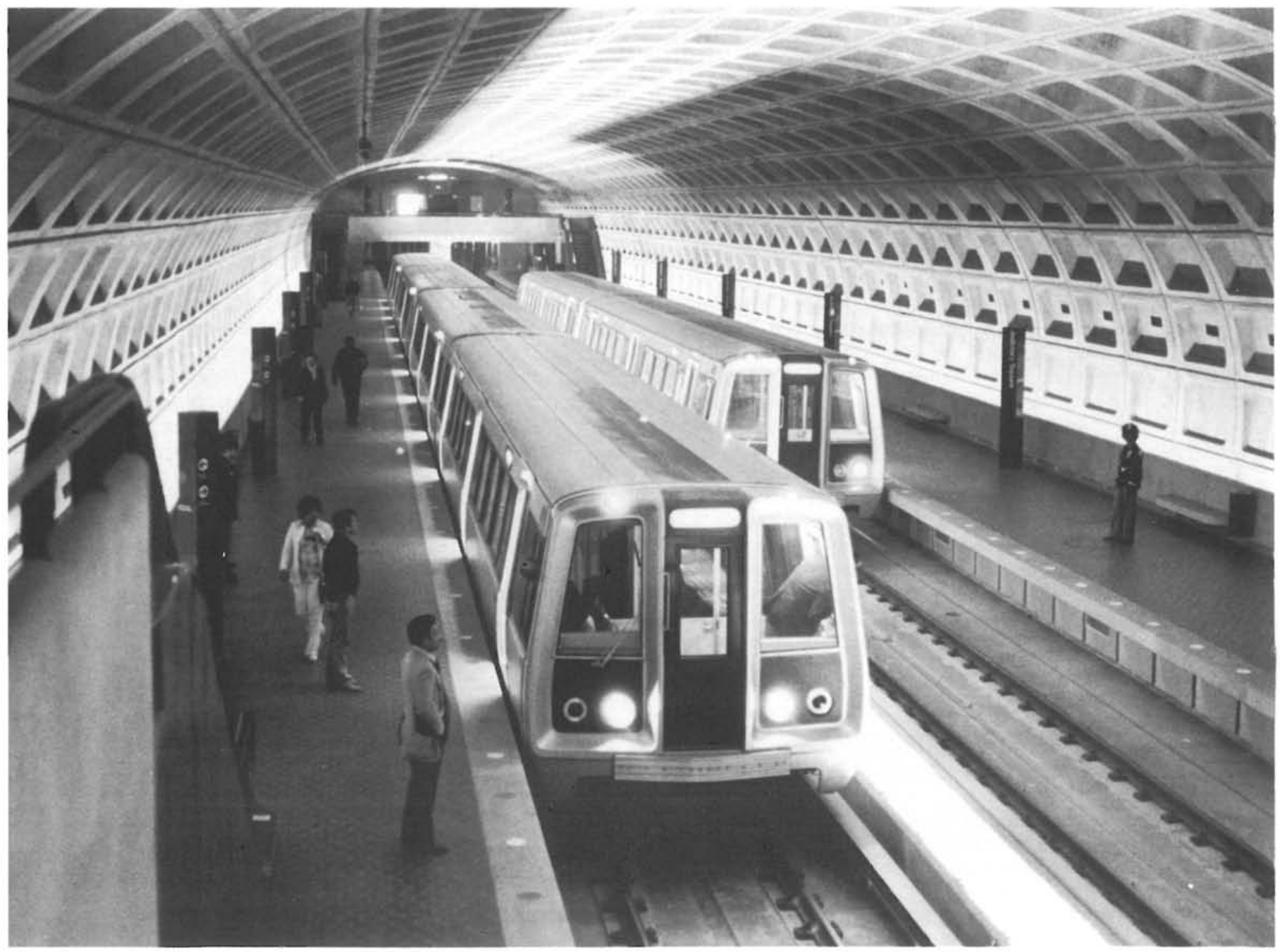


En sección transversal, la placa de la bóveda de 4 pulgadas $(101 \mathrm{~mm}$ ) de espesor tiene una doble configuración semicilíndrica apoyada en un pilar central. Cada placa doble cilíndrica está rigidizada longitudinalmente en dos vigas de $12 \times 10$ pulgadas $(305 \times 254 \mathrm{~mm})$ y, transversalmente a los pilares, por nervios de ancho de 1,5 pies $(457 \mathrm{~mm})$ con espesor variable. Las placas y vigas están postesadas sólo en la dirección longitudinal, y los nervios han sido postesados transversalmente.

\section{Concepto estructural}

La estructura de la vía se acomoda al empleo de carriles soldados de manera continua directamente a la superestructura. Los pasadores existentes en el soporte de la estructura limitan los movimientos térmicos en dirección longitudinal, debiendo además asegurar la contención lateral. Én consecuencia, las vigas curvas de las vías y sus pilares se han proyectado para resistir la resultante transversal de las fuerzas térmicas desarrolladas en cada uno de los pasadores directos de fijación, que están separados aproximadamente 30 pulgadas $(0,80 \mathrm{~m})$ de los centrales a lo largo de las vías curvas. El sistema estructural se ideó para que exista la mayor continuidad posible, manteniendo el movimiento logitudinal de la estructura dentro de los límites de tolerancia de los pasadores de los carriles. La construcción de pilares y vigas de vía integrales elimina alguna articulación en las intersecciones. Igualmente los cojinetes son ocultados a la vista en las proximidades de las juntas de expansión.

El uso de la superestructura continua de hormigón pretensado proporciona la oportunidad de proyectar elementos relativamente superficiales con pequeñas flexiones verticales. La sobrecarga teórica, peso propio y flexiones de deslizamiento son de menor magnitud que las contraflechas de compensación. Al eliminar la necesidad de las contraflechas la construcción se simplificó consiguiéndose un soporte de las vías más uniforme, contribuyendo de este modo a mejorar la calidad del recorrido. Este tipo de construcción facilita la introducción de transiciones elevadas $y$ en espiral necesarias en las curvas de las vías $y$, además, proporciona una transición más suave de la estructura dual de separación de vías a la disposición en caja doble $y$, análogamente, desde una superestructura de dos vigas a un sistema de tres vigas en las estaciones.

\section{Construcción de las vigas de las vías}

Diversos proyectos de premoldeado se propusieron para los tramos de las unidades continuas de doble espacio localizadas en el exterior de la estación aérea. Se eligió finalmente el moldeado in situ alternativo en toda la longitud de la estructura elevada.

Dos condiciones significativas se impusieron a la construcción de las vigas:

1. $\left.{ }^{a}\right)$ Para garantizar el adecuado espacio libre de las operaciones de alzado, fue obligatorio que las unidades de las vigas de las vías, a doble espacio, se colocarán y postesarán antes de la construcción de las pilas contiguas que soportan las ménsulas.

2. ${ }^{a}$ Las estructuras de apuntalamiento de los pilares de la ménsula, además de su cometido usual de soportar la discontinuidad del peso propio cerca de los pilares de los extremos durante el tesado, tienen otro que es el de ayudar a llevar a cabo el descimbramiento gradual y simultáneo para evitar la excesiva excentricidad del peso propio o impacto durante la transferencia de las reacciones verticales a los pilares de la ménsula.

El sistema VSL de pretensado fue el escogido, tensándose desde un solo extremo. Tres tubos de acero rígido galvanizado se instalaron en cada alma de las vigas-cajón del carril con cada conducto conteniendo de ocho a dieciocho cordones (diámetro, $12,7 \mathrm{~mm}$; resistencia, $19.000 \mathrm{~kg} / \mathrm{cm}$ ) de 7 alambres.

Se empleó una junta de construcción en los elementos del cajón justo debajo de la losa del tablero. Tiene la ventaja de asegurar el control cerrado de la colocación de la losa de la parte inferior. El apuntalamiento horizontal, consistente en vigas de ala ancha, soporta los moldes de la viga-cajón y tiene una serie de chapitas colocadas apropiadamente para permitir la flexión de los elementos provisionales de acero bajo el primer vertido.

Se colocaron torres de acero rebajado, prefabricado, debajo de las vigas de acero para formar, junto con el pilar central de hormigón previamente vertido, un sistema de cimbras a cuatro espacios bajo cada unidad continua de dos espacios. 
El segundo vertido se realizó por elementos de hormigón convencionalmente reforzado, comportándose como una estructura continua a cuatro espacios. Tal acción necesitó refuerzo adicional en las almas de la viga-cajón para acomodar la resultante de los esfuerzos de tracción desarrollados sobre los elementos provisionales. Por eso, durante el proceso de construcción, la viga de la vía se transformó desde una viga provisional continua a cuatro espacios, con elementos de hormigón convencionalmente armado, hasta una viga-cajón continua a dos espacios. Se tuvo especial consideración en el efecto de la recuperación elástica de la fuerte carga horizontal de los elementos de apuntalamiento, que tienden a evitar la acción del peso propio durante el pretensado.

\section{Puentes}

Para ver de qué forma los diferentes consultings de ingeniería resuelven los problemas de proyecto en los puentes de acero y estructuras aéreas, se comparan aquí dos entre los doce construidos:

- El puente que atraviesa el Pentágono, de 3.026 pies $(922,3 \mathrm{~m})$ de longitud, consta de 22 vanos con curvas opuestas en los extremos.

Las vigas de acero se han seleccionado para que actúen como conductos que resistan las cargas torsionales.

Tienen una estructura interna cruzada y una barra, en la parte superior, para mantener la forma del cajón y proporcionar rigidez torsional durante la construcción y hormigonado del tablero.

Se han usado dos tipos distintos de vigas-cajón: uno para el tramo recto, y otro para los tramos curvos.

Las vigas-cajón del tramo recto se proyectaron con almas verticales de diferente espesor y alas horizontales en la parte inferior. La otra viga-cajón, simétrica, se acomoda a la variación de peralte.

\section{FOTOS: PAUL MYATT}

STAN ALLAN

PHIL PORTLOCK

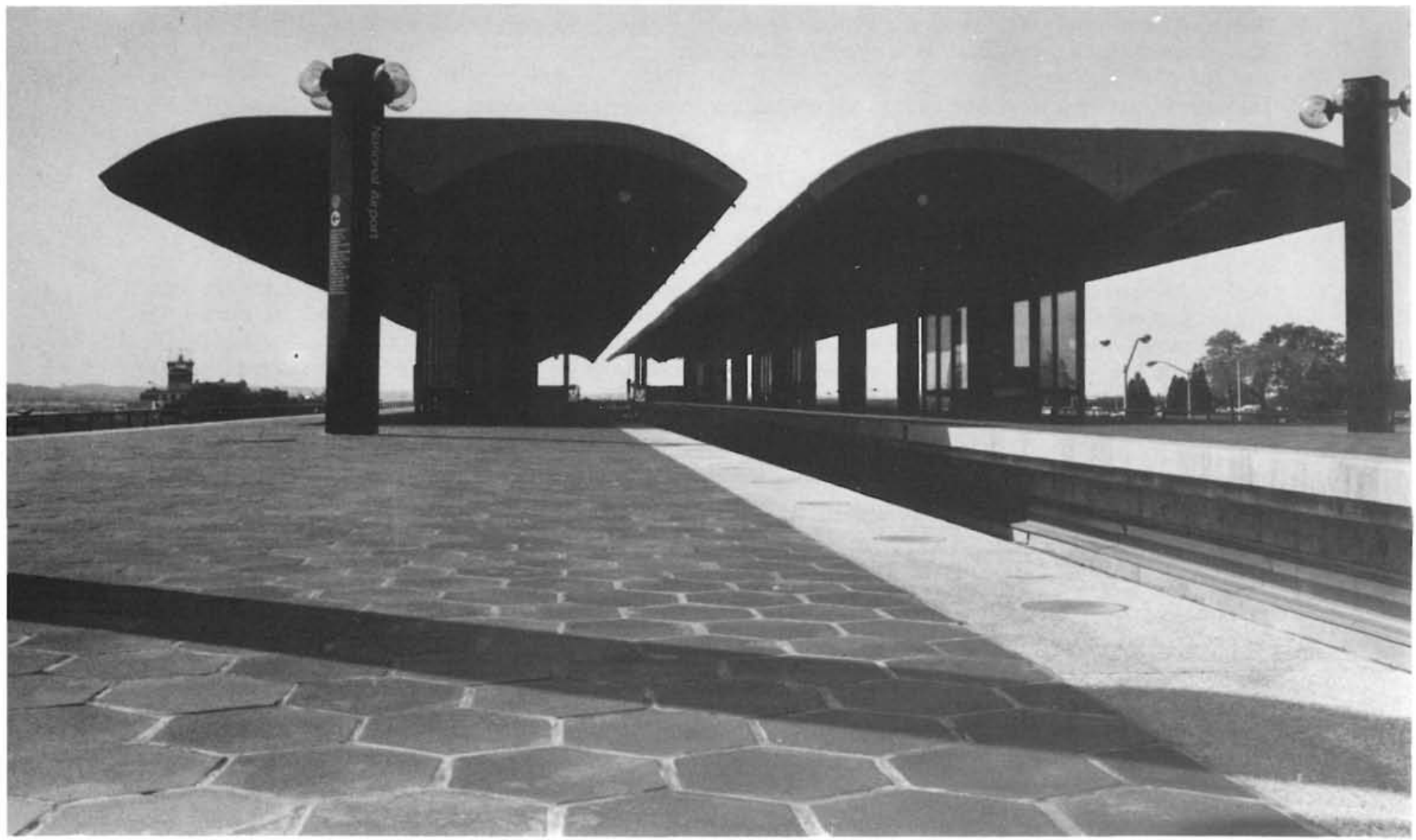


Aunque las vigas-cajón se hacen unidas, los tableros de las vigas se proyectaron como unidades separadas 0,75 pulgadas $(15 \mathrm{~mm})$, separación localizada a un lado del paseo de seguridad que corre por debajo de la mediana.

Se prestó especial importancia a la estética, en este proyecto, debido a encontrarse situado dentro de Washington. Las vigas-cajón se han hecho de acero meteorizado y pintadas en gris claro por el exterior para resaltar los colores de las estructuras cercanas, dejándose sin pintar los interiores.

- El puente que atraviesa el río Anacostia, de 7.645 pies $(2.330,2 \mathrm{~m})$, consta de 55 vanos y está realizado, en parte, sobre terraplén y, en parte, como estructura aérea.

Al igual que para el puente del Pentágono se escogió el acero para que resistiera mejor las cargas torsionales $y$, también, para que proporcionara una estructura que se mezclara agradablemente con el entorno. El acero meteorizado, hasta color bermejo profundo, minimiza los costos de mantenimiento y produce un costo razonable por unidad de tensión.

Existen, sin embargo, algunas diferencias con el proyecto del Pentágono:

1) En las curvas, las vigas-cajón empleadas en Anacostia son inclinadas por razones estéticas y para minimizar los costes de fabricación debido a la conservación del espesor del alma constante.

2) A causa de que es un perfil liso continuo, y con cara continua del alma del carril, las cabezas de los pilares de acero pudieron disimularse en el interior de las vigas.

3) Las dos vigas-cajón y sus tableros horizontales forman una unidad de composición; todos los cajones están interiormente armados.

Las vigas-cajón inclinadas a lo largo de las curvas poseen un problema potencial de fabricación porque la estructura de cajón forma una espiral cuando atraviesa una curva. Los ingenieros resolvieron esto por simulación de una espiral mediante una serie de curvas compuestas con cada tramo de viga-cajón, conteniendo dos secciones de diferente radio.

Otro problema es el efecto de rotación creado en los pilares con vanos de diferente longitud. Para minimizar estos esfuerzos de rotación se hacen ménsulas de diferentes longitudes en las cabezas de los pilares.

Ingenieros - De Leuw, Cather y Cía. (R. S. O'Neil, M. Asce, E. A. Fairhead).

\section{résumé \\ METRO DE WASHINGTON U.S.A.}

Harry Weese et associés, architects

Dans cet article sont décrits les travaux de la première phase du Métro de Washington qui, commencé en 1969, vera sa terminaison en 1983 et sera le type le plus moderne de train métropolitain de l'Amérique du Nord.

II est à deux voies et sera suffisant pour trois millions d'usagés.

Différents systèmes d'ouvrages ont été employés pour sa réalisation: tunnel artificie réalisé a l'aide d'une tranchée recouvert ensuite; tunnel perforé et structures aériennes.

Les caractéristiques des stations répondent aux plus grandes exigences, elles comprenaux plus grandes exigences, elles comprennent des circuits fermes de telévision, air conditione, systeme pour attenuer le bruit, le feu et control automatique du Métro.

Sont étudiés particulièrement les deux ponts sur le Pentagone et l'Anacostia, signalan leurs différences et, egalement, la structure aérienne de l'Aéroport National.

\section{summary UNDERGROUND RAILWAY IN WASHINGTON, U.S.A.}

Harry Weese and Associates, Architects

This article describes the works involved in the first stage of the Washington Underground (Subway) system which was begun in 1969 and scheduled for completion in 1983 and is the most modern metropolitan railway in North America.

Lines have double track and will carry three million passengers daily.

Different construction methods have been used throughout: tunnel formed by digging a trench then roofed and covered, excavated tunnel and elevated structures.

Stations features answer to the strictest demands, provided with closed circuit television, air conditioning, noise dampening systems, special access ways, fire protection systems and automatic traffic control.

Special attention is given to the two bridges over the Pentagon and over the Anacostia, pointing out their differences and the elevated structure at the National Airport.

\section{zusammenfassung \\ UNTERGRUNDBAHN VON \\ WASHINTON - USA \\ Harry Weese und Partner, Architekten}

In diesem Artikel werden die Arbeiten der ersten Etappe der Untergrundbahn von Washington beschrieben. Dieselben begannen im Jahre 1969 und bei deren Beendung im Jahre 1983 werden sie das modernste Untergrundbahnsystem von Nordamerika darsteilen.

Die U-Bahn ist doppelgleisig und für drei Millionen Benützer pro Tag geplant.

Bei der Durchführung sind verschiedene Bausysteme verwendet worden: künstlicher Tunnel
erstellt mittels Aushebung eines später abgeerstellt mittels Aushebung eines später abge-
deckten Grabens; Tunneldurchstich und Hochstrukturen.

Die Stationen genügen den höchsten Anforderungen, denn sie sind mit geschlossener Fernsehanlage, Klimaanlage, Schalldämpfersystem, speziellen Zugängen, Feuerschutzeinrichtung und automatischer U-Bahn-Kontrolle ausgestattet.

Es wird im besonderen auf die beiden Brücken über dem Pentagon und dem Anacostia unter Hinweis auf deren Unterschiede und auf die Hochstruktur des nationalen Lufthafens eingegangen. 